amount of data has been treated in precisely the same way. It would appear that such variations as may correlate with the moon are more apparent during some years than others. There is also some indication that there is a change of phase with lapse of time, so that the more years that are included in a single investigation for this effect the smaller is the resulting value.

An investigation by $\mathbf{M r}$. Kimura, appearing in the same number of the Proceedings as Mr. Kawasaki's paper, directs attention to the fact that if the declinations of the stars used in vol. 7 of the International Latitude Service are to be adjusted from the measures of latitude therein contained, a correction of $+0.11^{\prime \prime}$ is needed. While the addition of this amount to all the declinations necessarily increases the mean latitude of all the stations, Mr. Kimura finds the surprising fact that Mizusawa has apparently shifted south by so much as $0 \cdot 12^{\prime \prime}$ when the results of vol. 7 were compared with the results of vol. 5 .

It does not seem improbable that geophysical changes may be taking place which confuse the problem. In view of this, it would seem desirable to restrict investigations for these small variations of the moon's position to intervals of not more than three years at a time. Intercomparisons of the in. vestigations of these relatively short periods may lead to further light on the question.

In this connexion it should be mentioned that in the latest report of the Association de Géodésie de l'Union Géodésique et Géophysique Internationale, Dr. Walter D. Lambert has directed attention to the fact that Schumann published in $1906^{3}$ an investigation in which he found a large lunar term in latitude of the order of $0.03^{\prime \prime}$, which depended on the moon's hour angle and not twice the hour angle. Schumann's earlier work would appear reasonably consistent with my findings from investigations of observations for the years $1909,1910,1911$, and for Gaithersburg in 1913 and $1914^{4}$.

Cambridge, Massachusetts.

Harlan T. Stetson. April 3.

1 NATURE, 131, 437 (1933).

Proc. Imp. Acad., Tokyo, 11, No. 10 (1935).

Ergänzungshefte zu den Astronomischen Nachrichten No. 11. - NATURE, 1\%8, 127 (1929).

\section{Number of Ions produced in Dielectric Liquids by Cosmic Rays}

TrE electrical conductivity of liquid dielectrics in their normal state depends to a great degree on their purity. By a very careful purification, the conductivity of some liquids of this type can be lowered to about $10^{-18} \Omega^{-1} \mathrm{~cm} .^{-1}$.

It is significant that a further reduction of conductivity cannot be obtained by means of any of the known methods of purification. Investigations on the conduction of electricity through liquid dielectrics show the existence of a close analogy between the properties of these liquids and those of ionised gases ${ }^{1}$. The residual conductivity may be attributed to (1) impurities which cannot be removed, (2) a small conductivity of the character proper to metals or semi-conductors, (3) lowering of the barrier of potential on the surfaces of metallic electrodes under the influence of the contact with liquids ${ }^{2}$, (4) ionising action of cosmic rays. It may be noted that the ionising action of cosmic ray bursts in liquid dielectrics was studied recently by Prof. C. Białobrzeski and the author ${ }^{3}$.

The purpose of this note is to show that the cosmic rays are responsible for the conductivity observed. The calculations are based on the results of my measurements with hexane $\left(\mathrm{C}_{6} \mathrm{H}_{14}\right)$ filling a multiple parallel plate condenser. The volume of the liquid was 810 c.c. and the surface of the electrodes collecting the ions was $540 \mathrm{~cm}^{2}$. From the value of the saturation current, namely, to $10^{-16} \mathrm{amp} . / \mathrm{cm} .{ }^{2}$, it follows that the number of pairs of ions produced $420 \mathrm{~cm}^{3}$ sec. $^{-1}$.

If we suppose that the amount of ionisation by cosmic rays is proportional to the number of electrons in a given substance, we can easily calculate that the above number corresponds to 0.7 pair of ions per cubic centimetre per second in air at N.T.P. Taking the number of cosmic ray particles as given by Millikan to be $1.48 \pm 0.055 \mathrm{~cm}^{-2} \mathrm{~min} .^{-1}$, the ionising power of a cosmic ray particle amounts to $1.7 \times 10^{4}$ pairs of ions per cm. in hexane which corresponds to 28.6 ion pairs in air at normal conditions. This is rather smaller but of the same order as the number of ions produced by cosmic rays or obtained by various authors ${ }^{5}$. It therefore seems reasonable to conclude that the limit of conductivity of liquid dielectrics is nearly reached, and that the residual conductivity is due mainly to ionising action of cosmic rays.

The above remarks concern only the penetrating components of cosmic radiations, $A$ and $C$, according to the scheme of Geiger-Fünfer ${ }^{5}$; the secondary soft radiations, $D$ and $E$, produced by the hard radiations in the metal parts of the apparatus can be responsible only for surface effects appearing at the electrodes.

\section{ADAMCZEWSKr.}

Institute of Theoretical Physics,

Joseph Piłsudski University,

Warsaw. April 20.

1 I. Adamczewski, Acta Physica Polonica, 3, 235 (1934).

2 A. Nikuradse, "Das flüssige Dielektrikum". (Berlin: Springer, 1934.)

C. Białobrzeski and I. Adamczewski, NATURE, 136, 109 (1935).

I. Adamczewski, loc. cit. 1935.

H. Geiger, "Frgebnisse der exakten Naturwissenschaften", 14,

\section{Electrical Changes in Interfacial Films}

DuRING the course of an investigation of the ionic permeability of thin non-aqueous films, some interest. ing observations were made in regard to the electrical conductance of the films.

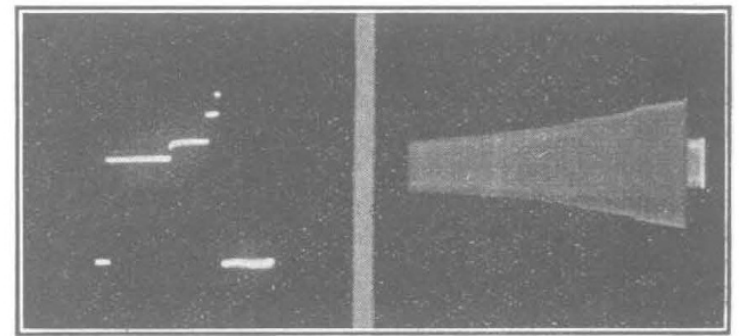

Fic. 1. Oscillograms on single bubble fllms of carbon tetrachloride Ordinates: Input potential (parallel with the conductance). Abscissæ: Time axis (to be read from the left, total duration about 1 second). Left-hand figure: $50 \mathrm{mv}$. D.c. in the bubble circuit. Right-hand flgure: constant $\mathbf{\Lambda . 0 .}, 1,000$ cycles, in the bubble circuit.

The arrangement was in principle as follows: Thin films of the non-squeous liquids were formed using a technique somewhat similar to the blowing of a soap bubble, the inside and outside air being replaced by aqueous electrolyte solutions in which 